\title{
Frequency-dependent viscous flow in channels with fractal rough surfaces
}

Andrea Cortis $^{\text {a) }}$ and James G. Berryman

Earth Sciences Division, Lawrence Berkeley National Laboratory, Berkeley, California 94720, USA

The viscous dynamic permeability of some fractal-like channels is studied. For our particular class of geometries, the ratio of the pore surface area-to-volume tends to $\infty$ (but has a finite cutoff), and the universal scaling of the dynamic permeability, $k(\omega)$, needs modification. We performed accurate numerical computations of $k(\omega)$ for channels characterized by deterministic fractal wall surfaces, for a broad range of fractal dimensions. The pertinent scaling model for $k(\omega)$ introduces explicitly the fractal dimension of the wall surface for a range of frequencies across the transition between viscous and inertia dominated regimes. The new model provides excellent agreement with our numerical simulations.

a) Electronic mail: acortis@ibl.gov 


\section{INTRODUCTION}

The dynamic interaction between a flowing fluid and the solid constituents of a porous medium is a key issue controlling wave propagation in geological ${ }^{1-3}$, biological $^{4,5}$, and engineered systems ${ }^{6,7}$. The general theory of wave propagation in porous media was developed in references ${ }^{8-12}$. In these theoretical developments, it is invariably assumed that it is always possible to identify two separate spatial scales, denoted by $x$ (the macroscopic, "slow" scale), and by $y$ (the microscopic, "fast" scale). When the characteristic length scale of the pores, $L_{y}$, is significantly smaller than the characteristic length of a macroscopic sample of the porous medium, $L_{x}$, i.e., $\epsilon=L_{y} / L_{x} \ll 1$, it is possible to uncouple elastic motion of solid matrix from oscillations of pore fluid ${ }^{13}$, and viscous from thermal dissipation ${ }^{14}$. In this work, we consider an isothermal system and assume that the driving force is a small-amplitude harmonic macroscopic pressure gradient $\nabla\langle p\rangle$ oscillating at a frequency $\omega$. The operator $\langle\cdot\rangle$ represents a spatial average of microscopic quantities over the fluid part of a porous medium with porosity, $\phi$. Assuming small-amplitude flow perturbations, it is possible to write all the quantities of interest in the form $f(\mathbf{r}, t)=\hat{f}(\mathbf{r}, \omega) \exp (i \omega t)$. Under such assumptions, a linear relationship exists between $\langle\mathbf{v}\rangle$ and $\nabla\langle p\rangle$, the coefficient of proportionality being a complex-valued function $k(\omega)$, called the viscous dynamic permeability.

Despite the great variability of the pore microgeometries considered in the literature ${ }^{15-18}$, it is generally found, both experimentally and numerically, that the normalized dynamic permeability $\tilde{k}(\omega)$ matches reasonably well a scaling function ${ }^{19,20}$ which depends on a characteristic frequency $\omega_{c}$ and a similarity parameter, $M$. Remarkably, $M$ is usually found to be almost configuration independent and close to unity, as long as the pore surface is locally smooth ${ }^{19,21,22}$. Experimental observations, however, indicate that pore roughness may exhibit self-similar characteristics over a wide range of length scales. Experimental studies based on different experimental techniques ${ }^{23-25}$ (e.g., scanning electron microscopy, small-angle neutron scattering, nitrogen adsorption isotherm) have demonstrated that, for instance, the pore space of sandstones and shales is approximately fractal over length scales ranging from $10 \AA$ to $100 \mu \mathrm{m}$, with corresponding fractal dimensions varying between 2.57 and 2.87. Other experimental studies ${ }^{26,27}$ have proven that fractured-rock wall surfaces also display fractal and multifractal properties over a range of scales between $0.1 \mu \mathrm{m}$ to $1 \mathrm{~cm}$.

Numerical investigations of the effects of fractal walls on the dynamic permeability have 
been the subject of previous works ${ }^{28-30}$. In this study, we explore the effects of such selfsimilar rough surfaces on the scaling of the dynamic permeability. Other studies have considered the problem of the static (frequency independent) flow in fractal trees ${ }^{31,32}$.

We present accurate numerical simulations of longitudinal frequency-dependent Stokes' flow in channels with fractal pore-surface structure, similar to the ones considered by Pozrikidis $^{30}$. The effect of self-similar sharp edges on the flow streamlines (transverse flow) will be considered separately elsewhere, as the corrections to the universal scaling associated with this type of singularity ${ }^{21,22}$ add-up in a non-trivial way to the effects which are the main object of study here.

In Section II we develop the theoretical basis of our analysis, which is followed, in Section III by a review of some basic concepts of fractal geometry. Section IV illustrates the numerical methods and the dynamic-response solutions for a broad range of fractal dimension, whereas Section V presents the modified scaling function that are used to model the numerical solutions. Finally, conclusions and open problems are summarized in Section VIII.

\section{THEORY}

\section{A. Homogenization of the flow equations}

The linearized fluid motion is fully characterized at the microscopic level by small perturbations to state of rest of the fluid velocity $\hat{\mathbf{v}}(\mathbf{r}, \omega)$, and fluid pressure $\hat{p}(\mathbf{r}, \omega)$. Expressing the pressure in units of $L_{x} \eta^{2} / \rho L_{y}^{3}$, and the frequency $\omega$ in units of $\nu / L_{y}^{2}$, the governing flow equations are the (nondimensional) frequency-dependent Stokes' equations ${ }^{13,14,16}$ :

$$
\begin{gathered}
i \omega \hat{\mathbf{v}}=-\epsilon^{-1} \nabla \hat{p}+\nabla^{2} \hat{\mathbf{v}}, \\
\nabla \cdot \hat{\mathbf{v}}=0, \\
\hat{\mathbf{v}}=0 \quad \text { on } A_{f s},
\end{gathered}
$$

where $A_{f s}$ is the fluid-solid surface.

Homogenization of these flow equations requires expressing $\hat{\mathbf{v}}$ and $\hat{p}$, and the differential 
operator $\nabla$ as a function of the slow scale $x$ and the fast scale $y$ (see Sanchez-Palencia ${ }^{33}$ ),

$$
\begin{aligned}
\hat{\mathbf{v}}(x, y) & =\hat{\mathbf{v}}_{0}(x, y)+\epsilon \hat{\mathbf{v}}_{1}(x, y)+\epsilon^{2} \hat{\mathbf{v}}_{2}(x, y)+\cdots \\
\hat{p}(x, y) & =\hat{p}_{0}(x, y)+\epsilon \hat{p}_{1}(x, y)+\epsilon^{2} \hat{p}_{2}(x, y)+\cdots \\
\nabla & =\epsilon \nabla_{x}+\nabla_{y} .
\end{aligned}
$$

Collecting the terms with the same power in $\epsilon$ leads to a hierarchy of equations:

$$
\begin{aligned}
& \nabla_{y} \hat{p}_{0}=0 \\
& i \omega \hat{\mathbf{v}}_{0}-\nabla_{y}^{2} \hat{\mathbf{v}}_{0}+\nabla_{y} \hat{p}_{1}=-\nabla_{x} \hat{p}_{0}, \\
& \nabla_{y} \cdot \hat{\mathbf{v}}_{0}=0 \\
& \left.\hat{\mathbf{v}}\right|_{A_{f s}}=0 .
\end{aligned}
$$

Equation (3a) indicates that $\hat{p}_{0}$ is a macroscopic variable, whereas equation ( $\left.3 \mathrm{~b}\right)$ shows that the fluid is incompressible at the microscopic scale. Since the fields $\hat{p}_{1}(x, y)$, and $\hat{\mathbf{v}}_{0}(x, y)$, are the solutions of linear systems, they can be formally expressed by means of linear operators acting on the macroscopic source term $-\nabla_{x} \hat{p}_{0}$ :

$$
\begin{aligned}
& -\hat{p}_{1}=\mathrm{P}_{\omega}(x, y) \cdot \nabla_{x} \hat{p}_{0}, \\
& -\hat{\mathbf{v}}_{0}=\mathrm{V}_{\omega}(x, y) \cdot \nabla_{x} \hat{p}_{0} .
\end{aligned}
$$

The linear operators $\mathrm{P}_{\omega}(x, y), \mathrm{V}_{\omega}(x, y)$ are vector and second rank tensor operators respectively. Substitution of equations (4) into (3) yields the following set of partial differential equations:

$$
\begin{aligned}
& i \omega \mathrm{V}_{\omega}-\nabla_{y}^{2} \mathrm{~V}_{\omega}+\nabla_{y} \mathrm{P}_{\omega}=\mathbf{I}, \\
& \nabla_{y} \cdot \mathrm{V}_{\omega}=0 \\
& \left.\mathrm{~V}_{\omega}\right|_{A_{f s}}=0
\end{aligned}
$$

where $\mathbf{I}$ is a second rank identity tensor. 


\section{B. Macroscopic flow equations}

Averaging of (4b) over the (fast) $y$-scale, leads to the macroscopic equation

$$
\left\langle\hat{\mathbf{v}}_{0}\right\rangle=-\frac{k(\omega)}{\phi} \cdot \nabla_{x} \hat{p}_{0}
$$

where

$$
k(\omega)=\phi\left\langle\mathrm{V}_{\omega}(x, y)\right\rangle
$$

which is an extension of the classical Darcy law in the frequency domain. Written in dimensional form, (6) reads

$$
\langle\hat{\mathbf{v}}\rangle=-\frac{k(\omega)}{\eta \phi} \nabla\langle\hat{p}\rangle
$$

The low frequency limit of the real part of the dynamic permeability, $k_{0}=\lim _{\omega \rightarrow 0} \operatorname{Re}(k(\omega))$, is the static Darcy permeability which can be calculated as, $k_{0}=\phi\left\langle\mathrm{V}_{0}\right\rangle L_{y}^{2}$, where $\mathrm{V}_{0}$ is the solution of (5) for a frequency $\omega=0$. To study the high frequency limit of (8), it is convenient to rewrite it in terms of Newton's law, by introducing a dynamic tortuosity, $\alpha(\omega)$, as follows:

$$
\rho \alpha(\omega) i \omega\langle\hat{\mathbf{v}}\rangle=-\nabla\langle\hat{p}\rangle .
$$

This formula results from Brown's work ${ }^{34}$, showing the connection between electrical and inertial properties.

The limit of $\alpha(\omega)$ for $\omega \rightarrow+\infty$, is called the tortuosity of the porous medium, $\alpha_{\infty}$, and equals $\alpha_{\infty}=\left\langle\mathrm{V}_{\infty}^{2}\right\rangle /\left\langle\mathrm{V}_{\infty}\right\rangle^{2}$. The velocity field $\mathrm{V}_{\infty}$ is the high-frequency limiting solution of (5) and can be expressed as the gradient of a harmonic potential field $\psi$, i.e., $\mathrm{V}_{\infty}=\nabla \psi$ and $\nabla^{2} \psi=0$ with homogeneous Neumann boundary conditions at the fluid-solid interface. As a first order approximation, the macroscopic law describing the fluid flow in the high frequency regime can be expressed via Newton's law of motion:

$$
i \omega \rho \alpha_{\infty}\langle\hat{\mathbf{v}}\rangle=-\nabla\langle\hat{p}\rangle
$$

The low frequency limit of the real part of $\alpha(\omega), \alpha_{0}=\lim _{\omega \rightarrow 0} \operatorname{Re}[\alpha(\omega)]$, is called static viscous tortuosity, and can be computed as ${ }^{35-37} \alpha_{0}=\left\langle\mathrm{V}_{0}{ }^{2}\right\rangle /\left\langle\mathrm{V}_{0}\right\rangle^{2}$, where $\mathrm{V}_{0}$ is the solution of (5) at $\omega=0$. Substituting the Poiseuille flow solution into the above equation, we find that for 
a straight tube (3D flow) $\alpha_{0}=4 / 3$, while for a set of parallel planes ( $2 \mathrm{D}$ flow) $\alpha_{0}=6 / 5$. From (8) and (9), we derive the relation between the dynamic parameters $k(\omega)$ and $\alpha(\omega)$

$$
\frac{\alpha(\omega)}{\alpha_{\infty}}=\frac{k_{0}}{k(\omega)} \frac{\omega_{c}}{i \omega}
$$

where $\tilde{\omega}=\omega / \omega_{c}$, and $\omega_{c}=\frac{\eta \phi}{\alpha_{\infty} k_{0} \rho}$, is the characteristic frequency for the transition between the viscous and the inertia dominated regimes. For smooth pore-wall surfaces, the asymptotic high-frequency behavior for $\alpha(\omega)$ is ${ }^{19}$

$$
\alpha(\omega) \sim \alpha_{\infty}\left[1+(1-i) \frac{\delta(\omega)}{\Lambda}\right]
$$

where

$$
\delta(\omega)=(2 \eta / \rho \omega)^{1 / 2},
$$

is the viscous boundary layer thickness, which can take on arbitrarily small values as frequency increases. The $\Lambda$ parameter is a characteristic viscous length scale defined as a velocity-weighted pore volume $\left(V_{f}\right)$-to-pore surface $\left(A_{f_{s}}\right)$ ratio:

$$
\Lambda=2 \frac{\int_{V_{f}} \mathrm{~V}_{\infty}^{2} d V}{\int_{A_{f s}} \mathrm{~V}_{\infty}^{2} d A}
$$

and is therefore a purely geometrical parameter. The boundary layer thickness, $\delta(\omega)$, in $(13)$ identifies a boundary layer region where, as a consequence of the no-slip boundary condition, energy is dissipated by viscous forces, whereas in the complementary region, the fluid flow becomes inviscid (inertial response only).

The high-frequency asymptotic behavior of $k(\omega)$ is given by

$$
\frac{k(\omega)}{k_{0}} \sim-i \tilde{\omega}^{-1}+\frac{1}{2}(1+i) \sqrt{M} \tilde{\omega}^{-\frac{3}{2}}
$$

where

$$
M=\frac{8 k_{0} \alpha_{\infty}}{\phi \Lambda^{2}}
$$

is a nondimensional scaling parameter of order one ${ }^{19,22}$. The $M$ parameter enters also the 
higher-order terms of the low-frequency asymptotic behavior of $\alpha(\omega)$

$$
\alpha(\omega) \sim \alpha_{0}-i\left(\alpha_{\infty} \tilde{\omega}^{-1}-\frac{2}{M} \frac{\left(\alpha_{\infty}-\alpha_{0}\right)^{3}}{\alpha_{\infty}^{2}} \tilde{\omega}\right)
$$

Finally, we note that the drag force, $f(\omega)$, is proportional to the time derivative of the added $\operatorname{mass}^{20}\left(\alpha(\omega) / \alpha_{\infty}-1\right)$ :

$$
f(\omega)=-i \tilde{\omega} \alpha_{\infty}\left(\frac{\alpha(\omega)}{\alpha_{\infty}}-1\right)
$$

For smooth pore-walls, the dynamic permeability (and consequently the dynamic tortuosity, and the complex-valued drag force) exhibit a universal scaling behavior. The universal scaling can be modeled in terms of a function, $F(\omega)$, which is defined in such a way to satisfy both the low- and high-frequency theoretical limits, and to be exact in the limiting case of $2 \mathrm{D}$ slit flow. The expression for the dynamic permeability reads thus ${ }^{19,20}$

$$
\frac{k(\omega)}{k_{0}}=[F(\omega)+i \tilde{\omega}]^{-1}
$$

where

$$
F(\omega)=1-\frac{3}{10} \frac{M}{a}\left(1-i \tilde{\omega} \frac{1}{3} b \frac{\tanh (z)}{z-\tanh (z)}\right)
$$

and

$$
\begin{aligned}
a & =\frac{\alpha_{0}-1}{\alpha_{\infty}}, \\
b & =50 \frac{a^{2}}{M}, \\
z & =\sqrt{b i \tilde{\omega}} .
\end{aligned}
$$

The parameter $z$, scales like the inverse of the thickness of the boundary layer, $z \sim \delta^{-1}$ : we will use this fact to generalize the scaling functions to the case of fractures with fractal surfaces.

\section{FRACTAL WALL SURFACES}

Let us consider rock fractures delimited by two rough surface walls symmetric with respect to a horizontal plane, and analyze the effects that asperities (at many scales, down to some pre-microscopic cutoff) have on frequency-dependent flow. We assume that the 
fracture is periodic in the plane that contains the fracture aperture, and that there is no geometry variation in the direction perpendicular to this plane. Moreover, we assume that the flow direction is also directed perpendicularly to the fracture aperture plane. This flow configuration can then be studied there by means of a two-dimensional model in which the only pertinent component of the velocity vector is along the longitudinal direction $x_{3}$, i.e., $v_{x_{1}}=v_{x_{2}}=0$, where $x_{i}$, with $i=1,2,3$ represent the three spatial coordinates in a rectangular (orthogonal) coordinate system. The longitudinal component $v_{x_{3}}$, is a function only of the two spatial coordinates of the longitudinal cross-section, $x_{1}, x_{2}$, hence $\frac{\partial v_{x_{1}}}{\partial x_{3}}=0$, from which it follows that the condition on the divergence of the flow is automatically satisfied. Moreover, a pressure gradient exists only along the longitudinal direction $x_{3}$, from which it follows that the Stokes problem reduces to a Poisson problem in the scalar variable $v_{x_{3}}$. The corresponding closure problem reduces thus to finding $\mathrm{V}_{\omega}^{(3)}$, which is the $x_{3}$ component of the closure problem vector $\mathrm{V}_{\omega}=\left(\mathrm{V}_{\omega}^{(1)}, \mathrm{V}_{\omega}^{(2)}, \mathrm{V}_{\omega}^{(3)}\right)$, satisfying

$$
i \omega \mathrm{V}_{\omega}^{(3)}-\nabla_{y}^{2} \mathrm{~V}_{\omega}^{(3)}=1, \quad \text { and }\left.\quad \mathrm{V}_{\omega}^{(3)}\right|_{A_{f s}}=0
$$

For such a geometry, $\alpha_{\infty} \equiv 1$, i.e., there is no tortuosity along the flow streamlines. Furthermore, the expression for the characteristic viscous length $\Lambda$ reduces to ${ }^{14}$

$$
\Lambda=2 \frac{V_{f}}{A_{f s}}
$$

Before analyzing the precise geometry of these surfaces, it may be useful to recall a few definitions from Euclidean and fractal geometry. The Euclidean measure of a $d$-sphere ${ }^{38}$ with, $d \in \mathbb{N}$, is equal to $b_{d}=\frac{\pi^{d / 2}}{\Gamma(d / 2+1)}$. Rescaling a $d$-sphere by a factor $\lambda$, results in a measure proportional to $\lambda^{d}$. The exponent of the scaling factor in the rescaled measure is called the dimension of the object. Fractal geometrical objects generalize these notions by allowing non-integer dimensions. This type of scaling is commonly observed in many natural phenomena. Natural fracture-wall surfaces, for instance, present asperities at different scales, and there is ample consensus in the rock mechanics literature that rock surfaces are characterized by non-integer dimensions over a broad range of spatial scales, typically from $10^{-5}-10^{1}$ meters. Single fractures wall surfaces are fractal therefore in a stochastic sense.

In this work, we will consider only fracture-wall surfaces defined as the Cartesian product 
of a deterministic fractal $K$ and a 1-d segment of arbitrary length. The dimension of the fractal curve, $1<d<2$, characterizes completely the dimension of the fractal curve. The deterministic fractal considered in this study is a Köch fractal curve ${ }^{39}$. The prototypical Köch curve is generated by dividing the segment $[0,1]$ into three smaller segments of equal length $l_{1}=1 / 3$, and replacing the central segment with two segments of length $l_{1}$ to form an equilateral triangle. This construction is then repeated over the resulting segments, and iterated indefinitely. The resulting curve, $K$, is autosimilar, i.e., admits a partition $\left\{K_{i}\right\}, i=1, \ldots, n$, such that every $K_{i}$ is homothetical (identical when rescaled) to $K$. After $k$ iterations the Euclidean length of the curve built over the unit segment equals $L_{k}=(4 / 3)^{k}$ a value which tends to $\infty$ for $k \rightarrow \infty$. The area of the corresponding fracture-wall surface tends, therefore, also to $\infty$.

Let $L_{d}(K)$ be the $d$-dimensional measure of $K$. There exists at most one value of $d$ for which $0<L_{d}(K)<\infty$ and we indicate this number as $d$, the Hausdorff dimension ${ }^{39}$ of $K$. The measure of the Köch curve $K_{[0,1]}$ constructed on the unit segment $L=1[\mathrm{~m}]$, is

$$
L_{d}\left(K_{[0,1]}\right)=b_{d} \sum_{i}^{4^{n}}\left(\operatorname{diam}\left(K_{i}\right) / 2\right)^{d}=b_{d} \frac{1}{2^{d}}\left(\frac{4}{3^{d}}\right)^{n}=b_{d} \frac{1}{2^{d}}=0.953097\left[\mathrm{~m}^{\frac{\log 4}{\log 3}}\right]
$$

a value that does not depend on the iteration $n$ in the construction of the fractal curve.

Real world materials, however, do not of course exhibit such ideal autosimilar behavior at all scales of observation. Rather, they show upper and lower cutoff lengths at which the autosimilar property breaks off. Below the lower cutoff length, and above the upper cutoff length, the geometry of the object is Euclidean, i.e., measures scale with integer exponents. Any approximation of such a real world object will entail a finite number $n$ of iterations of the omothetical construction. The cutoff length scale, $\ell$, equals the smallest of the geometric details of the fracture surface.

In channels with fractal pore-fluid surfaces $A_{f s}$, the classical scaling in (20) does not hold anymore because $\Lambda \equiv 2 V_{f} / A_{f s} \rightarrow 0$, and consequently $M \rightarrow \infty$. If the yardstick of our fractal analysis is represented by the thickness of the boundary layer, $\delta$, we expect that the measure of the boundary layer itself will roughly follow the scaling of the fractal surface. At low frequencies, the boundary layer will be sufficiently separated from the actual fractal surface and thus will be smooth, with a length roughly proportional to $L_{1}=4 / 3[\mathrm{~m}]$. At very high frequencies, when $\delta \ll \ell$, the finest detail of the surface for a given maximum 
number of iterations $n$, the measure of the boundary layer will scale again as an Euclidean geometrical object. For the classical Köch curve of dimension $d=\log 4 / \log 3$, the Euclidean length of the boundary layer at such high frequencies will be thus roughly proportional to $L_{n}=(4 / 3)^{n}[\mathrm{~m}]$, which for large $n$ is a large number, but not infinite. There must exist, therefore, an intermediate range of frequencies over which the length of the boundary layer scales with a non-integer exponent, and takes values of a length to a non-integer power. In this range of frequencies, the scaling of the boundary layer thickness must be different from the classical expression $\delta \sim \tilde{\omega}^{-1 / 2}$.

To fix ideas, consider a fracture with permeability $k_{0}=1.59 \times 10^{-8} \mathrm{~m}^{2}$, tortuosity $\alpha_{\infty}=1$, fractal dimension $d \sim 1.27$, and water as the oscillating fluid (at a temperature $\mathrm{T}=25{ }^{\circ} \mathrm{C}$, water density $\rho=997 \mathrm{~kg} \cdot \mathrm{m}^{-3}$, and viscosity $\left.\eta=8.90 \times 10^{-4} \mathrm{~Pa} \cdot \mathrm{s}\right)$. The corresponding viscous-inertia transition frequency is $\omega_{c}=63 \mathrm{~Hz}$. A range of nondimensional frequencies between $10^{-2}<\tilde{\omega}<10^{4}$ corresponds therefore to dimensional frequencies roughly in the range between $1 \mathrm{~Hz}$ and $0.1 \mathrm{GHz}$. As will be shown in our numerical computations section, the dimension of the corresponding computational cell is on the order of $1 \mathrm{~mm}$. For a maximum number of iterations $n=5$, the length of the smallest detail of the surface is roughly $\ell \sim\left(1 \mathrm{~mm} / 4^{n}\right) \sim 1 \mu \mathrm{m}$, a value which is within the range of previous experimental determinations of the fractal dimensions for single fractures surface walls.

Pozrikidis ${ }^{30}$ also introduces another scaling exponent for fractal geometries, $d^{\prime}$, the gain in wall length with increasing refinement. The values of $d^{\prime}$ corresponding to the fractal dimensions considered in this study can be found in Table III.

It becomes clear therefore that the scaling expression for the viscous boundary layer presented in (13), and the associated scaling models presented in Section II B need to be modified. The numerical simulations of the dynamic permeability presented in the next Section will determine the analytical expression of the scaling models appropriate for fractal surface channels.

\section{NUMERICAL SIMULATIONS}

In this Section, we present a series of numerical solutions of the closure problem (24) for a two-dimensional cross-section with fractal walls defined by Köch-like curves for a range of fractal dimension. Kostek et al. ${ }^{29}$ and Pozrikidis ${ }^{30}$ studied longitudinal and transverse 
oscillating flow for such geometries. The fractal-walls profiles in our study (see Figure 1) were generated by means of a simple L-system algorithm ${ }^{40}$. We allowed the fractal dimension $d$ of the surface to vary in the range $1.16<d<1.49$. We constructed two sets of geometries, for two different values of the maximum number of iterations in the fractal construction, $n=4$ and $n=5$. A periodic repetition of a unit cell of length 1 and width 1 is assumed along the transverse direction. We exploit the reflection symmetry of unit cell along the middle plane. Equation (24) is solved numerically for different values of the nondimensional frequency $\tilde{\omega}$ by means of a finite element numerical scheme; a typical mesh is shown in Figure 2. From the numerical solutions of (24), we calculate the dynamic permeability, $k(\omega)$, as a function of frequency, and the scaling functions coefficients, $\alpha_{0}, \alpha_{\infty}$, and $\Lambda$.

In Figure 3, we plot the evolution of the value of the Darcy (static) permeability, $k_{0}$ as a function of the porosity for a range of maximum iterations in the fractal geometry construction, $n$. It is observed that, in this range of porosity variation, the permeability follows closely a Kozeny-Carman relationship, $k_{0} / \phi=c_{1} \frac{1}{180} \frac{\phi^{2}}{(1-\phi)^{2}}+c_{2}$. The linear regression lines are shown in red, and the corresponding coefficients are listed in Table I. As the value of the permeability remains bounded, and the value of $\Lambda \rightarrow 0$, it follows that the scaling parameter, $M \rightarrow \infty$.

In Figure 4, we present the evolution of the velocity field for different values of the frequency. At small frequencies, the phase of the velocity field, $\Theta\left(\mathrm{V}_{\omega}^{(3)}\right)$, (right hand side panels) is negligibly small, but the shape of the boundary layer can be appreciated from the plot of the absolute value of the velocity itself. At intermediate frequencies, we see that the boundary layer follows closely the profile of the fluid-solid interface, and as such exhibits the same fractal dimension scaling. At much higher frequencies, the boundary layer is indistinguishable from the bounding surface itself, and the phase of the velocity field is equal to $-\pi / 2$ throughout the fluid space, indicating an inertia-dominated flow regime. For these frequencies, the thickness of the boundary layer is much smaller than the smallest details of the pore-fluid surface, $\ell$; hence, the boundary layer thickness scales according to (13). This result can be better appreciated in Figure 5, which shows a contour plot of the boundary thickness as a function of the frequency. The precise position of the boundary layer is largely a matter of convention in the choice of the threshold. In this work, we define the boundary layer as the loci of the points for which $\Theta\left(\bigvee_{\omega}^{(3)}\right)=-0.7$. Similar results are obtained for different choices of the threshold. 
In Figure 6 we plot the evolution of the length and thickness of the boundary layer for the entire range of fractal dimensions, and for a number of iterations in the construction of the fractal $n=4$ (top panels), and $n=5$ (bottom panels). We observe that the length of the boundary layer, increases as the frequency increases. Moreover the rate of this increase is larger for larger values of the fractal dimension, $d$. The boundary layer length tends to stabilize to the Euclidean measure of the fracture surface, and this value increases with $d$. This stabilization of the value of the length happens above some critical frequency threshold, the value of which decreases with increasing $d$. We also note that the critical frequencies for the stabilization of the boundary layer length increase with the value of $n$. This can be understood by keeping in mind that the yardstick for the evolution of this length is the boundary layer thickness, $\delta$, represented in the left hand side panels of Figure 6: so, the higher the frequency, the smaller the thickness of the boundary layer, and when this thickness becomes smaller than the smallest segment length in the fractal curve, the solid surface which is subject to the viscous flow dissipation becomes flat for all intents and purposes. The red lines in Figure 6 indicate the regions over which it was possible to define a power law scaling for the boundary layer length and thickness. The critical frequency, $\tilde{\omega}^{\prime}$, and the corresponding exponents $\beta_{L}$, and $\beta_{\delta}$, respectively, are reported in Table II. The scaling exponent $\beta_{L}$, does not seem to correspond directly to the exponent $d^{\prime}$, the gain in wall length with increasing refinement. The exponent $\beta_{\delta}$ seems to maintain a value close to $-1 / 2$ only for $n=4$, whereas its values deviate significantly for $n=5$, showing a decrease for increasing, $d$.

We can now plot the numerical results of the dynamic response and the corresponding models. In Figure 9, we plot the frequency dependence of the added mass phase, $\Theta\left(\alpha(\omega) / \alpha_{\infty}-1\right)$. This representation enhances the differences between the numerical results and the scaling function. The blue curve in Figure 9, represents the model in 20, for a value of $\Lambda$ corresponding to the Euclidean length of the fractal curve, $L_{n}$. Such scaling function does not represent a good model of the dynamic tortuosity for these geometries. We will discuss the model represented by red lines in the next Section. 


\section{SCALING MODELS FOR FRACTAL MEDIA}

Johnson et al. ${ }^{19}$ proposed that the characteristic viscous length for fractal media, $\Lambda$, scales as $2 / \Lambda \sim \delta^{2-d_{e}}$, where $1<d_{e}<d$, is an exponent characterizing the dynamic process. Furthermore, these authors ${ }^{19}$ conjectured that for high frequencies, the added mass scales according to the expression

$$
\frac{\tilde{\alpha}(\omega)}{\alpha_{\infty}}-1 \sim\left(\frac{1}{2}(1-i) \sqrt{\frac{M_{e}}{\tilde{\omega}}}\right)^{\left(2-d_{e}\right)}
$$

where $M_{e}$, is a nondimensional effective scaling parameter. Our calculations show that, in the case of fractures with fractal pore walls, the added mass does not scale according to the power-law expression in (27). In Figure 7 , we plot the real and imaginary parts of the added mass as a function of the nondimensional frequency (in double-logarithmic scale) for the range of fractal dimensions analyzed in this work. While the real part follows a power-law best for $d<1.3$, the imaginary part does not follow the simple scaling in (27) for any value of the fractal dimension.

Pozrikidis $^{30}$ proposed that, in fractal media, the absolute value of the drag force, $f(\omega)$, scales as a function of the inverse boundary layer thickness, $\delta$, i.e.,

$$
|f(\omega)| \sim\left(\frac{1}{\sqrt{\tilde{\omega}}}\right)^{1+d^{\prime}}
$$

where $d^{\prime}$ is the gain in wall length with increasing refinement, and $\delta \sim \frac{1}{\sqrt{\tilde{\omega}}}$. The left hand side panel of Figure 8, shows the quantity $\left|\tilde{\omega}^{-1 / 2}\right| f(\omega)$ vs. $\tilde{\omega}^{-1 / 2}$ on a double-logarithmic scale. We estimated the value of the slope in the region where the graph can be approximated by a power-law. The right-hand side of Figure 8, shows the estimated slopes versus the value of $d^{\prime}$, and also displays a quadratic polynomial relationship between these two quantities. We cannot therefore confirm the Pozrikidis' claim ${ }^{30}$ that “... results for longitudinal motion provide an explicit example in which the magnitude of the drag force and rate of dissipation diverge according to the fractal dimension of the surface."

Instead, as it will be shown below, we find that the drag force scaling depends explicitly on the value of the fractal dimension $d$ in a well-contrained range of frequencies.

We start from the observation that there must exist a range of frequencies, $\tilde{\omega}_{l}<\tilde{\omega}<\tilde{\omega}_{u}$, 
for which the scaling of the boundary layer thickness does not follow the classical scaling (13). Outside of this range of frequencies, the dynamic process develops as if the surface were smooth, hence with no exponent corrections. The plots in Figure 6 show the evolution of the length and thickness of the boundary layer as a function of frequency, for $n=4$ and $n=5$.

Therefore, we propose to modify the expression for the $z$ nondimensional variable in $(23)$ as

$$
z=\left(g \frac{M(\omega)}{m^{2}} 3 i \tilde{\omega}\right)^{\gamma(\tilde{\omega})}
$$

where

$$
\begin{aligned}
\gamma(\tilde{\omega}) & =1 / 2, \quad \tilde{\omega}<\tilde{\omega}_{l} \\
\frac{1}{\left(\tilde{\omega}_{u}-\tilde{\omega}_{l}\right)} \int_{\tilde{\omega}_{l}}^{\tilde{\omega}_{u}} \gamma(\tilde{\omega}) d \tilde{\omega} & =1-\frac{d}{2}, \quad \tilde{\omega}_{l}<\tilde{\omega}<\tilde{\omega}_{u} \\
\gamma(\tilde{\omega}) & =1 / 2, \quad \tilde{\omega}>\tilde{\omega}_{u}
\end{aligned}
$$

i.e., we impose the condition that the average value of the correction in the range of frequencies $\tilde{\omega}_{l}<\tilde{\omega}<\tilde{\omega}_{u}$, to be dictated by the value of the fractal dimensions itself, $d$. The universal scaling parameter, $M$, is defined now as a function of the frequency, $M(\omega)$, as

$$
\begin{array}{cc}
M_{d}=\frac{8 k_{0}^{d} \alpha_{\infty}}{\phi L_{d}^{2}}, & \tilde{\omega}<\tilde{\omega}_{M} \\
M=\frac{8 k_{0} \alpha_{\infty}}{\phi \Lambda^{2}}, & \tilde{\omega}>\tilde{\omega}_{M},
\end{array}
$$

where the relevant characteristic viscous length for $\tilde{\omega}<\tilde{\omega}_{M}$ is given by the Hausdorff length of fractal curve, $L_{d}$. The two values of $M$ are interpolated by means of a $5^{\text {th }}$ degree polynomial.

Figure 9 shows the best-fit scaling (red lines) obtained with the modifications in Equations (29), (30), and(31) for the phase of the added mass. The proposed model captures all the essential features of the numerical data, with only three free parameters: the lower and upper cutoff frequencies, $\tilde{\omega}_{l}$ and $\tilde{\omega}_{u}$, and the transition frequency $\tilde{\omega}_{M}$. We conclude that the modified scaling introduced in (29), which introduces explicitly the value of fractal dimension of the surface, $d$, is the key to understand the frequency dependence of the dynamic response of oscillating fluids in porous media. 
As a concluding remark, we note that in Eq. (31), the expression in $M_{d}$ does not have exactly the same functional form as $M$ as the definition of the characteristic length for $d>1$ is not a ratio of volume over surface area. Should the characteristic viscous length be defined as volume to surface-area ratio, i.e., equal to $2 V_{f} / L_{d}$, then the pertinent exponent in (31) would be $2-d$, and not $d$. Nonetheless, for $d=1$, the numerical values and physical dimensions of $L_{d}$ and $\Lambda$, do coincide, and the smooth porous media model is recovered as a limiting case. To resolve this apparent inconsistency, various modifications of the expressions in (29), (30), and (31), aimed at introducing explicitly the characteristic viscous length as a volume to surface-area ratio, have been tested. Even though these modified expressions reproduce the numerical data with an accuracy comparable to the best-fits reproduced in Figure 9, they invariably require the introduction of an additional free-parameter in place of the fractal dimension exponent in (29). In other words, the explicit appearance of the fractal dimension value has disappeared from these modified models.

We have opted, therefore, for a model that reproduces the data with the minimum number of parameters, in this case Equations (29), (30), and(31), which invokes only three free parameters. The resulting apparent paradox will perhaps be resolved through a careful first-principles analysis of the boundary-layer scaling near the fractal surface. The classical expression for the velocity of a fluid oscillating on a flat surface (Rayleigh problem) is $v=v_{0} e^{-\zeta / \delta(\omega)} e^{i(\zeta / \delta(\omega)-\omega t)}$, with $v_{0}$ the reference velocity, and $\zeta$ a coordinate orthogonal to the oscillating flow direction. This expression is the solution to the diffusion equation $\partial v / \partial t=\nu \partial^{2} v / \partial x^{2}$. The profile of the oscillating velocity near a flat boundary is thus the solution to a classical diffusion problem, and is characterized by an exponential decay of the velocity amplitude, i.e., by a distribution with finite mean and variance.

The fractal surface, however, introduces anomalies in the diffusion process which cannot be described any longer by Gaussian distributions of the microscopic quantities ${ }^{41}$, but rather by Levy-type distributions, distributions characterized by infinite mean and variance. We speculate that the lack of a characteristic length scale into the fractal surface may be the equivalent of the lack of a mean value and variance in Levy-type distributions. The introduction of Levy distribution in the description of diffusion processes often translates in the introduction of convolution operators in space and time ${ }^{42}$. As such, we expect that the classical expression for the characteristic viscous length in (14), $\Lambda$, motivated by the solution of a potential flow (diffusion) problem, needs modification. A more detailed study 
of the viscous boundary-layer around fractal surfaces will be the object of a future study.

\section{CONCLUSIONS}

We have presented a numerical study on the effect of fractal wall surfaces on oscillatory fluid flow. Motivated by our interest in fluid flow in fractures, we have considered two dimensional longitudinal flows in channels whose side-wall geometries are bounded by deterministic fractal curves. For these geometries, the classical models valid for smooth wall surfaces break down. We proposed a modified scaling model for macroscopic quantities such as the dynamic permeability and tortuosity. These new models introduce explicitly the value of the fractal dimension of the surface as an independent parameter, and modify the classical expressions only in the range of frequencies where the boundary layer explores the fine structure of the fractal surface. The model proposed in this study has three free parameters, namely the lower and upper cutoff frequencies, $\tilde{\omega}_{l}$ and $\tilde{\omega}_{u}$, respectively, and a characteristic frequency for the transition to the high-frequency smooth-surface dynamic regime, $\tilde{\omega}_{M}$. Our model gives excellent agreement with the numerical simulations. Additional effort, however, is needed to provide a first-principle derivation of these modified scalings to include explicitly, via fractional-order derivatives, the anomalous nature of the diffusive processes in the viscous boundary layer. Finally, our results indicate that the fractal dimension of a fracture surface wall may be assessed by studying the high-frequency behavior of the added-mass phase signal in laboratory experiments.

\section{ACKNOWLEDGMENTS}

Work of both A.C. and J.G.B. was performed under the auspices of the U.S. Department of Energy, at the Lawrence Berkeley National Laboratory under Contract No. DE-AC0205CH11231. Support was provided specifically by the Office of Basic Energy Sciences, Division of Chemical Sciences, Geosciences, and Biosciences.

\section{REFERENCES}

${ }^{1}$ R. N. Chandler and D. L. Johnson, "The equivalence of quasistatic flow in fluid-saturated porous-media and Biot's slow-wave in the limit of zero frequency," Journal of Applied 
Physics 52, 3391-3395 (1981).

${ }^{2}$ O. Kelder and D. M. J. Smeulders, "Observation of the Biot slow wave in water-saturated Nivelsteiner sandstone," Geophysics 62, 1794-1796 (1997).

${ }^{3}$ D. M. J. Smeulders and M. E. H. VanDongen, "Wave propagation in porous media containing a dilute gas-liquid mixture: Theory and experiments," Journal of Fluid Mechanics 343, 351-373 (1997).

${ }^{4}$ S. S. Kraman, "Speed of low-frequency sound through lungs of normal men," Journal of Applied Physiology 55, 1862-1867 (1983).

${ }^{5}$ T. Yamamoto, T. Otani, H. Hagino, H. Katagiri, T. Okano, I. Mano, and R. Teshima, "Measurement of human trabecular bone by novel ultrasonic bone densitometry based on fast and slow waves," Osteoporosis International 20, 1215-1224 (2009).

${ }^{6}$ C. Perrot, R. Panneton, and X. Olny, "Computation of the dynamic thermal dissipation properties of porous media by Brownian motion simulation: Application to an open-cell aluminum foam," Journal of Applied Physics 102, 074917-1-13 (2007).

${ }^{7}$ C. Perrot, F. Chevillotte, and R. Panneton, "Dynamic viscous permeability of an opencell aluminum foam: Computations versus experiments," Journal of Applied Physics 103, 024909-1-8 (2008).

${ }^{8}$ C. Zwikker and C.W. Kosten, Sound Absorbing Materials (Elsevier Publishing Company, New York, 1949).

${ }^{9}$ M. A. Biot, "Theory of propagation of elastic waves in a fluid-saturated porous solid. I. Low-frequency range," J. Acoust. Soc. Am. 28, No.2, 168-178 (1956).

${ }^{10} \mathrm{M}$. A. Biot, "Theory of propagation of elastic waves in a fluid-saturated porous solid. II. Higher-frequency range," J. Acoust. Soc. Am. 28, No.2, 179-191 (1956).

${ }^{11}$ T. Lévy, "Propagation of waves in a fluid-saturated porous elastic solid," Int. J. Engng. Sci 17, 1005-1014 (1979).

${ }^{12}$ J. L. Auriault, "Dynamic behaviour of a porous medium saturated with a Newtonian fluid," Int. J. Engng. Sci. 18, 775-785 (1980).

${ }^{13}$ R. Burridge and J. B. Keller, "Poroelasiticity equations derived from microstructure," J. Acoust. Soc. Am. 70(4), 1140-1146 (1981).

${ }^{14}$ D. Lafarge, P. Lemarinier, and J.F. Allard, "Dynamic compressibility of air in porous structures at audible frequencies," J. Acoust. Soc. Am. 102 (1997). 
${ }^{15}$ J.-L. Auriault, L. Borne, and R. Chambon, "Dynamics of porous saturated media, checking of the generalized law of Darcy," J. Acoust. Soc. Am. 77 (5), 1641-1650 (1985).

${ }^{16} \mathrm{M}$. Zhou and P. Sheng, "First-principles calculations of dynamic permeability in porous media," Phys. Rev. B 39, No 16, 12027-12039 (1989).

${ }^{17}$ A.M. Chapman and J.J.L. Higdon, "Oscillatory Stokes flow in periodic porous media," Phys. Fluids A 4 (10), 2099-2116 (1992).

${ }^{18} \mathrm{Y}$. Achdou and M. Avellaneda, "Influence of pore roughness and pore-size dispersion in estimating the permeability of a porous medium from electrical measurements," Phys. Fluids A 4 (12), 2561-2673 (1992).

${ }^{19}$ D. L. Johnson, J. Koplik, and R. Dashen, "Theory of dynamic permeability and tortuosity in fluid-saturated porous media," J. Fluid Mech. 176, 379-402 (1987).

${ }^{20}$ S. R. Pride, F. D. Morgan, and A. F. Gangi, "Drag forces of porous-medium acoustics," Phys. Rev. B 47, No 9, 4964-4978 (1993).

${ }^{21}$ A. Cortis and D. M. J. Smeulders, "On the viscous length scale of wedge-shaped porous media," Int. J. Engng. Sci. 39, 951-962 (2001).

${ }^{22}$ A. Cortis, D. M. J. Smeulders, J.-L. Guermond, and D. Lafarge, "Influence of pore roughness on high-frequency permeability," Phys. Fluids 15, 1766-1775 (2003).

${ }^{23}$ A. J. Katz and A. H. Thompson, "Fractal sandstone pores - implications for conductivity and pore formation," Physical Review Letters 54, 1325-1328 (1985).

${ }^{24}$ A. P. Radliński, E. Z. Radlińska, M. Agamalian, G. D. Wignall, P. Lindner, and O. G. Randl, "Fractal geometry of rocks," Phys. Rev. Lett. 82, 3078-3081 (1999).

${ }^{25}$ Jian Ma, Hao Qi, and Po-zen Wong, "Experimental study of multilayer adsorption on fractal surfaces in porous media," Phys. Rev. E 59, 2049-2059 (1999).

${ }^{26}$ S. L. Huang, S. M. Oelfke, and R. C. Speck, "Applicability of fractal characterization and modeling to rock joint profiles," International Journal of Rock Mechanics and Mining Sciences \& Geomechanics Abstracts 29, 89-98 (1992).

${ }^{27}$ H. P. Xie, J. A. Wang, and E. Stein, "Direct fractal measurement and multifractal properties of fracture surfaces," Phys. Lett. A 242, 41-50 (1998).

${ }^{28}$ Donald L. Koch, "Attenuation of a compressional sound wave in the presence of a fractal boundary," Physics of Fluids 30, 2922-2927 (1987).

${ }^{29}$ S. Kostek, L. M. Schwartz, and D. L. Johnson, "Fluid permeability in porous media: Comparison of electrical estimates with hydrodynamical calculations," Phys. Rev. B 45, 
186-195 (1992).

${ }^{30}$ C. Pozrikidis, "Unsteady viscous-flow over irregular boundaries," J. Fluid Mech. 255, 1134 (1993).

${ }^{31} \mathrm{P} . \mathrm{Xu}$ and Yu. B., "The scaling laws of transport properties for fractal-like tree networks," J. Appl. Phys. 100, 1-8 (2006).

${ }^{32}$ P. Xu, Yu. B., Y. Feng, and M. Zou, "Permeability of the fractal disk-shaped branched network with tortuosity effect," Phys. Fluids 18, 1-4 (2006).

${ }^{33}$ E. Sanchez-Palencia, Non-Homogenoeus Media and Vibration Theory, Lecture Notes in Physics (Springer-Verlag, Berlin, 1980).

${ }^{34}$ R. J. S. Brown, "Connection between formation factor for electrical-resistivity and fluidsolid coupling factor in Biot equations for acoustic-waves in fluid-filled porous-media," Geophysics 45, 1269-1275 (1980).

${ }^{35}$ A. N. Norris, "On the viscodynamic operator in Biot's equations of poroelasticity," J. Wave Mater. Inter. 1 (4), 365-380 (1986).

${ }^{36} \mathrm{D}$. Lafarge, Propagation du son dans les matériaux poreux à structure rigide saturés par un fluide viscothermique: Définition de paramètres géométriques, analogie electromagnétique, temps de relaxation, Ph.D. thesis, Université du Maine, Le Mans, France (1993).

${ }^{37}$ A. Cortis, D. M. J. Smeulders, D. Lafarge, M. Firdaouss, and J.-L. Guermond, "Geometry effects on sound in porous media," in Proc. IUTAM Symposium on Theoretical and Numerical Methods in Continuum Mechanics of Porous Media. University of Stuttgart, Germany, September 5-10, 1999, Solid Mechanics and its applications, Vol. 87, edited by W. Ehlers (Kluwer, Dordrecht, 2001) pp. 187-192.

${ }^{38} \mathrm{~A} 1$-sphere is defined as the open segment $]-1,1\left[, b_{d}=2[\mathrm{~m}]\right.$. Analogously, the 2-sphere is the open circle of radius $1, b_{d}=\pi\left[\mathrm{m}^{2}\right]$ and the 3 -sphere is the open sphere of radius 1 , $b_{3}=\frac{4}{3} \pi\left[\mathrm{m}^{3}\right]$.

${ }^{39}$ B.B. Mandelbrot, The Fractal Geometry of Nature (Freeman and Company, New York, 1983).

${ }^{40}$ Przemyslaw Prusinkiewicz and Aristid Lindenmayer, The Algorithmic Beauty of Plants (Springer-Verlag, New York, 1990).

${ }^{41}$ Z. Wang, "Anomalous diffusion in fractal porous medium," Applied Mathemaics and Mechanics (English Edition) 21(10), 1145-1152 (2000). 
${ }^{42}$ B. Berkowitz, A. Cortis, M. Dentz, , and H. Scher, "Modeling non-fickian transport in geological formations as a continuous time random walk," Reviews of Geophysics 44, RG2003, doi:10.1029/2005RG000178 (2006). 


\begin{tabular}{|c|c|c|}
\hline$d$ & $c_{1}$ & $c_{2}$ \\
\hline 1.1646 & $2.6480 \times 10^{-2}$ & $4.0459 \times 10^{-2}$ \\
1.2002 & $3.1389 \times 10^{-2}$ & $3.5982 \times 10^{-2}$ \\
1.2359 & $3.4851 \times 10^{-2}$ & $3.2047 \times 10^{-2}$ \\
1.2715 & $3.6724 \times 10^{-2}$ & $2.8553 \times 10^{-2}$ \\
1.3071 & $3.6941 \times 10^{-2}$ & $2.5447 \times 10^{-2}$ \\
1.3427 & $3.5548 \times 10^{-2}$ & $2.2652 \times 10^{-2}$ \\
1.3783 & $3.2691 \times 10^{-2}$ & $2.0180 \times 10^{-2}$ \\
1.4139 & $2.8508 \times 10^{-2}$ & $1.7936 \times 10^{-2}$ \\
1.4496 & $2.3311 \times 10^{-2}$ & $1.5927 \times 10^{-2}$ \\
1.4852 & $1.7501 \times 10^{-2}$ & $1.4146 \times 10^{-2}$ \\
\hline
\end{tabular}

TABLE I. Linear Regression coefficients for the Kozeny-Carman relationship $k_{0} / \phi=c_{1} \frac{1}{180} \frac{\phi^{2}}{(1-\phi)^{2}}+$ $c_{2}$ for the range of fractal dimensions, $d$, considered in this study.

\begin{tabular}{|c|c|c|c|c|c|c|c|}
\hline & & \multicolumn{3}{|c|}{$n=4$} & \multicolumn{3}{|c|}{$n=5$} \\
\hline$d$ & $d^{\prime}$ & $\log _{10} \tilde{\omega}^{\prime}$ & $\beta_{L}$ & $\beta_{\delta}$ & $\log _{10} \tilde{\omega}^{\prime}$ & $\beta_{L}$ & $\beta_{\delta}$ \\
\hline 1.1646 & -0.1646 & 3.5862 & 0.0636 & -0.4953 & 3.5862 & 0.0494 & -0.4724 \\
\hline 1.2002 & -0.2002 & 3.3793 & 0.0829 & -0.4940 & 3.3793 & 0.0677 & -0.4698 \\
\hline 1.2359 & -0.2357 & 3.1724 & 0.1079 & -0.4958 & 3.1724 & 0.0856 & -0.4649 \\
\hline 1.2715 & -0.2714 & 2.9655 & 0.1330 & -0.4967 & 2.9655 & 0.1016 & -0.4569 \\
\hline 1.307 & -0.3069 & 2.7586 & 0.1584 & -0.4962 & 2.7586 & 0.1217 & -0.4522 \\
\hline 1.3427 & -0.3427 & 2.5517 & 0.1832 & -0.4922 & 2.5517 & 0.1488 & -0.4526 \\
\hline 1.3783 & -0.3780 & 2.3448 & 0.2052 & -0.4827 & 2.3448 & 0.1757 & -0.4491 \\
\hline 1.413 & -0.4137 & 2.1379 & 0.2274 & -0.4711 & 2.1379 & 0.2008 & -0.4363 \\
\hline 1.4496 & -0.4495 & 2.1379 & 0.2760 & -0.4927 & 2.1379 & 0.2138 & -0.4106 \\
\hline 1.4852 & -0.4852 & 2.1379 & 0.3302 & -0.5210 & 2.1379 & 0.2189 & -0.3788 \\
\hline
\end{tabular}

TABLE II. Exponents of the power law scalings for boundary layer length $\beta_{L}$ and thickness $\beta_{\delta}$, for $n=4$ and $n=5$. The value of $\log _{10} \bar{\omega}$ represents the limit over which a power law scaling could be estimated. These power law scalings are illustrated in red in Figure 6. 


\begin{tabular}{|c|c|c|c|c|c|c|c||c|c|c|}
\hline$d$ & $d^{\prime}$ & $k_{0} / \phi$ & $h_{e}$ & $\Lambda$ & $L_{d}$ & $M$ & $M_{d}$ & $\log _{10} \tilde{\omega}_{l}$ & $\log _{10} \tilde{\omega}_{M}$ & $\log _{10} \tilde{\omega}_{u}$ \\
\hline 1.1646 & -0.1646 & 0.0462 & 0.7449 & 0.4187 & 0.9744 & 2.1101 & 0.2349 & -0.9023 & 0.8858 & 3.8438 \\
1.2002 & -0.2002 & 0.0416 & 0.7122 & 0.3530 & 0.9681 & 2.6704 & 0.1879 & -1.0435 & 0.3336 & 3.5595 \\
1.2359 & -0.2357 & 0.0374 & 0.6815 & 0.2982 & 0.9617 & 3.3624 & 0.1489 & -1.2152 & 0.2824 & 3.4936 \\
1.2715 & -0.2714 & 0.0335 & 0.6526 & 0.2523 & 0.9550 & 4.2121 & 0.1169 & -1.2658 & 0.0843 & 3.2849 \\
1.3071 & -0.3069 & 0.0300 & 0.6255 & 0.2140 & 0.9482 & 5.2387 & 0.0910 & -1.4271 & -0.1251 & 3.1232 \\
1.3427 & -0.3427 & 0.0268 & 0.6001 & 0.1819 & 0.9411 & 6.4760 & 0.0700 & -1.4910 & -0.1299 & 3.0020 \\
1.3783 & -0.3780 & 0.0239 & 0.5767 & 0.1554 & 0.9339 & 7.9178 & 0.0534 & -1.6503 & -0.2671 & 2.8815 \\
1.4139 & -0.4137 & 0.0212 & 0.5551 & 0.1330 & 0.9264 & 9.6030 & 0.0402 & -1.8443 & -0.3926 & 2.8166 \\
1.4496 & -0.4495 & 0.0188 & 0.5357 & 0.1144 & 0.9188 & 11.5032 & 0.0299 & -1.9293 & -0.3870 & 2.7321 \\
1.4852 & -0.4852 & 0.0167 & 0.5191 & 0.0992 & 0.9110 & 13.5533 & 0.0220 & -2.0602 & -0.4532 & 2.6512 \\
\hline
\end{tabular}

TABLE III. Summary of the macroscopic parameters for the various simulations for $n=4$.

\begin{tabular}{|c|c|c|c|c|c|c|c||c|r|r|}
\hline$d$ & $d^{\prime}$ & $k_{0} / \phi$ & $h_{e}$ & $\Lambda$ & $L_{d}$ & $M$ & $M_{d}$ & $\log _{10} \tilde{\omega}_{l}$ & $\log _{10} \tilde{\omega}_{M}$ & $\log _{10} \tilde{\omega}_{u}$ \\
\hline 1.1646 & -0.1646 & 0.0461 & 0.7433 & 0.3488 & 0.9744 & 3.0309 & 0.2341 & -1.0620 & $>4$ & 5.1885 \\
1.2002 & -0.2002 & 0.0414 & 0.7101 & 0.2826 & 0.9681 & 4.1507 & 0.1870 & -1.2249 & $>4$ & 5.0718 \\
1.2359 & -0.2357 & 0.0372 & 0.6789 & 0.2293 & 0.9617 & 5.6587 & 0.1481 & -1.2278 & 1.8168 & 4.0376 \\
1.2715 & -0.2714 & 0.0333 & 0.6493 & 0.1863 & 0.9550 & 7.6820 & 0.1161 & -1.3006 & 1.7147 & 3.9823 \\
1.3071 & -0.3069 & 0.0298 & 0.6215 & 0.1518 & 0.9482 & 10.3600 & 0.0902 & -1.4749 & 1.6248 & 3.9554 \\
1.3427 & -0.3427 & 0.0266 & 0.5952 & 0.1237 & 0.9411 & 13.9075 & 0.0694 & -1.6573 & 1.4817 & 3.9038 \\
1.3783 & -0.3780 & 0.0237 & 0.5709 & 0.1014 & 0.9339 & 18.4652 & 0.0528 & -1.8443 & 1.3054 & 3.8315 \\
1.4139 & -0.4137 & 0.0211 & 0.5483 & 0.0832 & 0.9264 & 24.3616 & 0.0397 & -1.9134 & 1.1087 & 3.6952 \\
1.4496 & -0.4495 & 0.0187 & 0.5278 & 0.0686 & 0.9188 & 31.7466 & 0.0295 & -2.0219 & 0.9693 & 3.5969 \\
1.4852 & -0.4852 & 0.0165 & 0.5102 & 0.0570 & 0.9110 & 40.6032 & 0.0217 & -2.1908 & 0.8385 & 3.5462 \\
\hline
\end{tabular}

TABLE IV. Summary of the macroscopic parameters for the various simulations for $n=5$. 

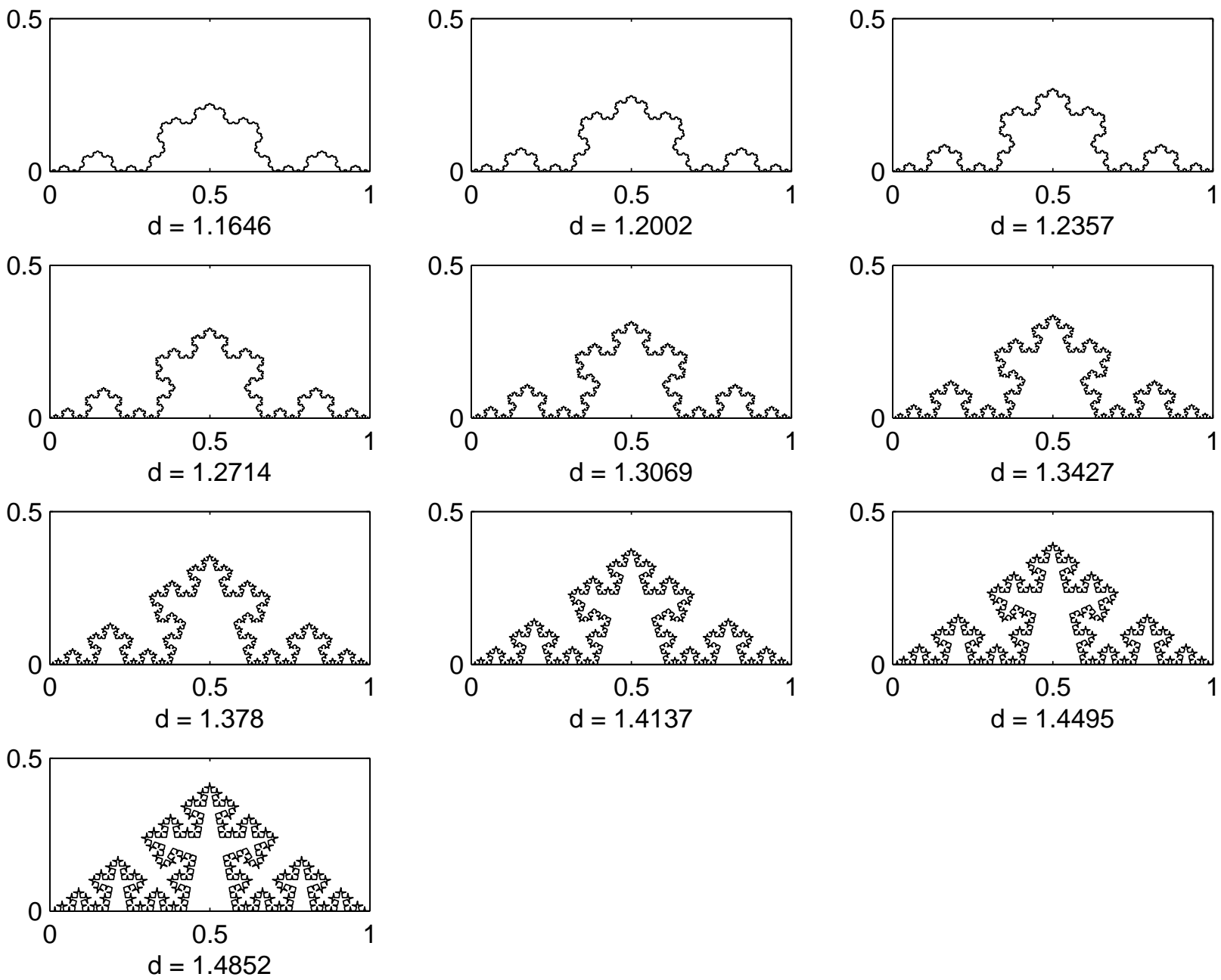

FIG. 1. Fractal surface profiles considered in this study. Only the bottom half of the computational cell is represented. 


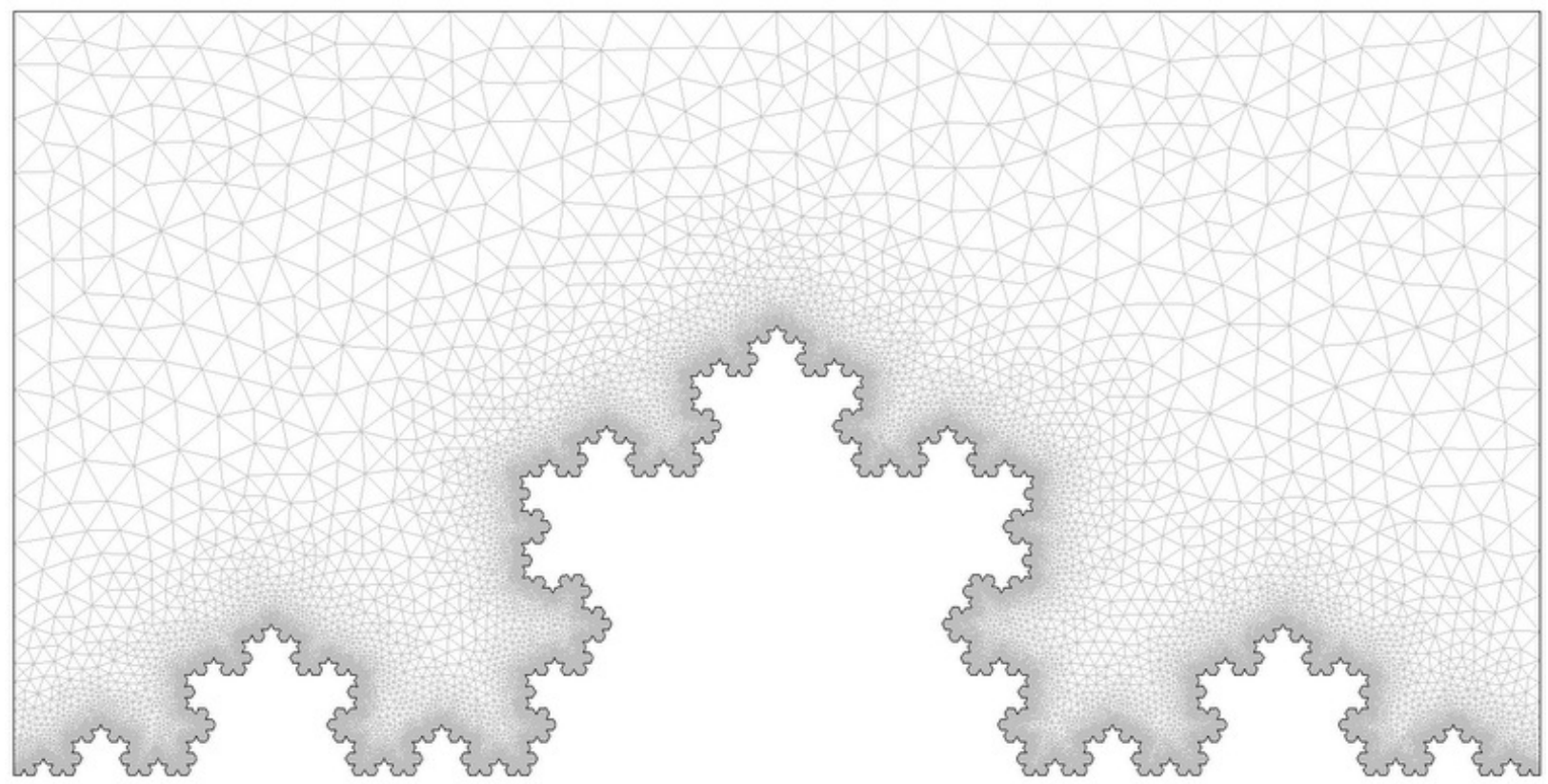

FIG. 2. Typical mesh used for the numerical simulations. Fractal dimension of the channel, $d=1.2714$. 


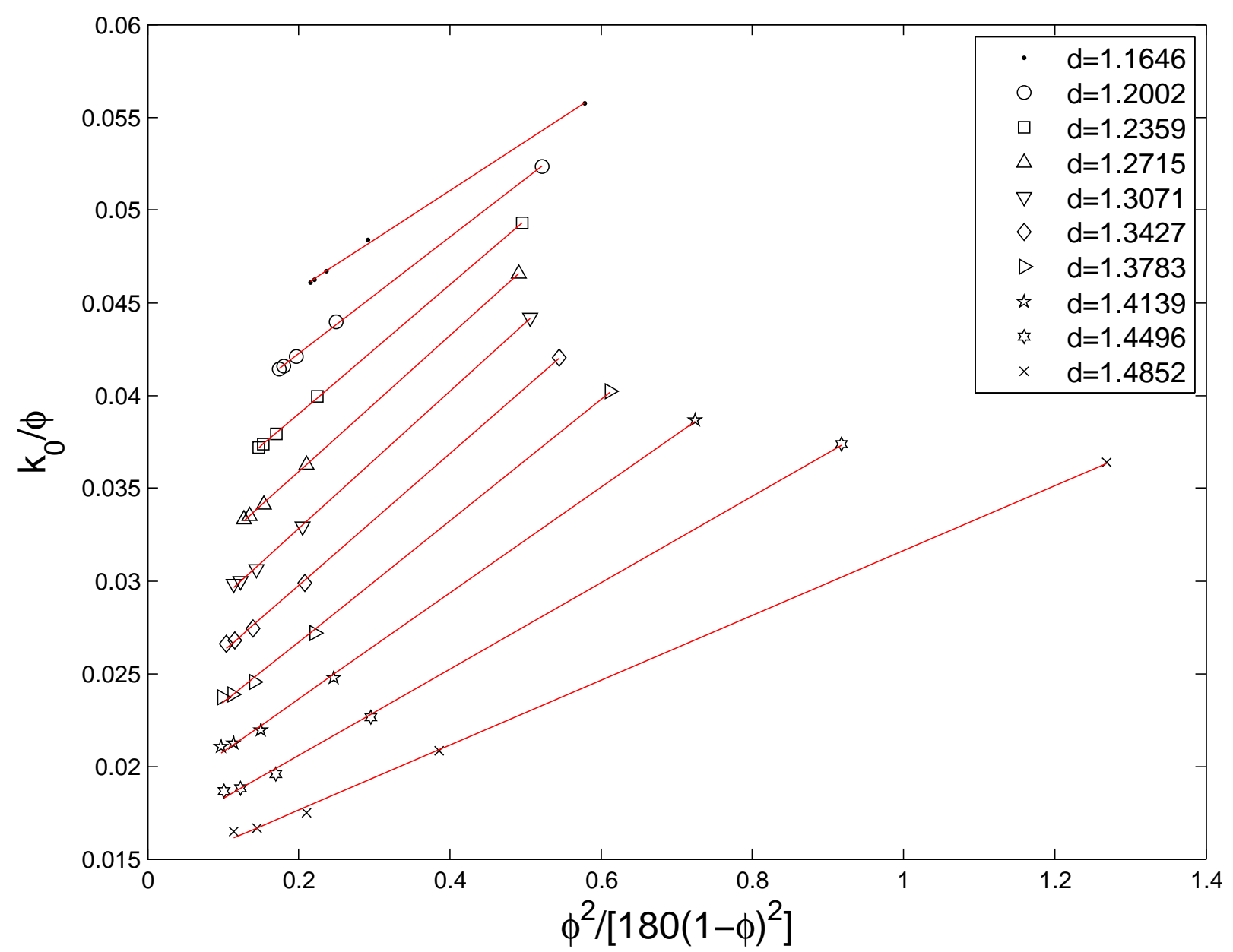

FIG. 3. Evolution of the Darcy (static) permeability, $k_{0}$, as a function of the porosity, $\phi$, at different values of the iteration, $n$. Linear regression lines are shown in red and the corresponding coefficients are listed in Table I. 


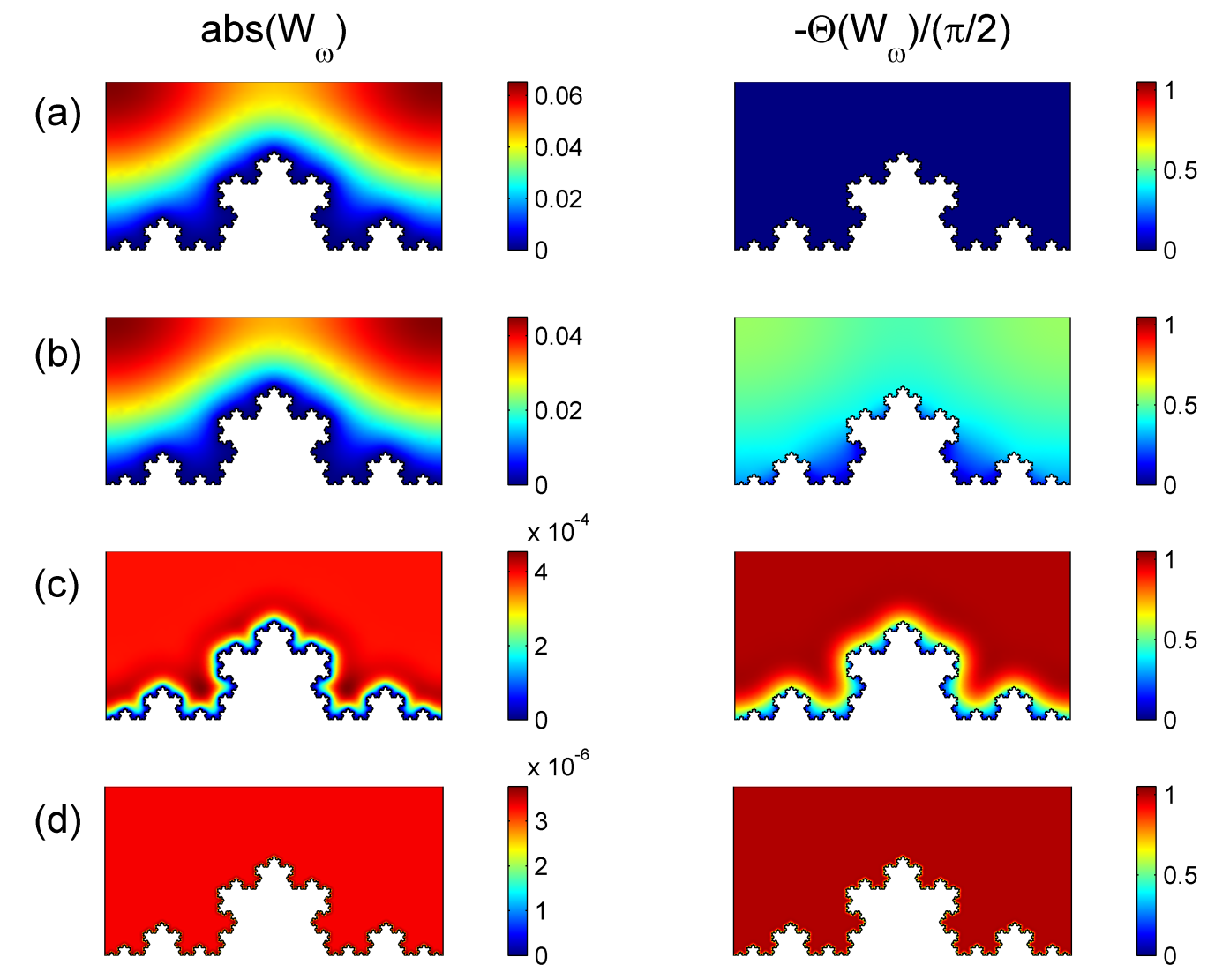

FIG. 4. Absolute value and phase of the velocity field $\mathrm{V}_{\omega}^{(3)}$ as a function of the frequency, $\tilde{\omega}$, for a fractured channel with fractal dimension $d=1.2714$, and a number of iterations $n=5$. (a) $\tilde{\omega}=0.01$; (b) $\tilde{\omega}=0.73$; (c) $\tilde{\omega}=85.31$; (d) $\tilde{\omega}=10000$. 


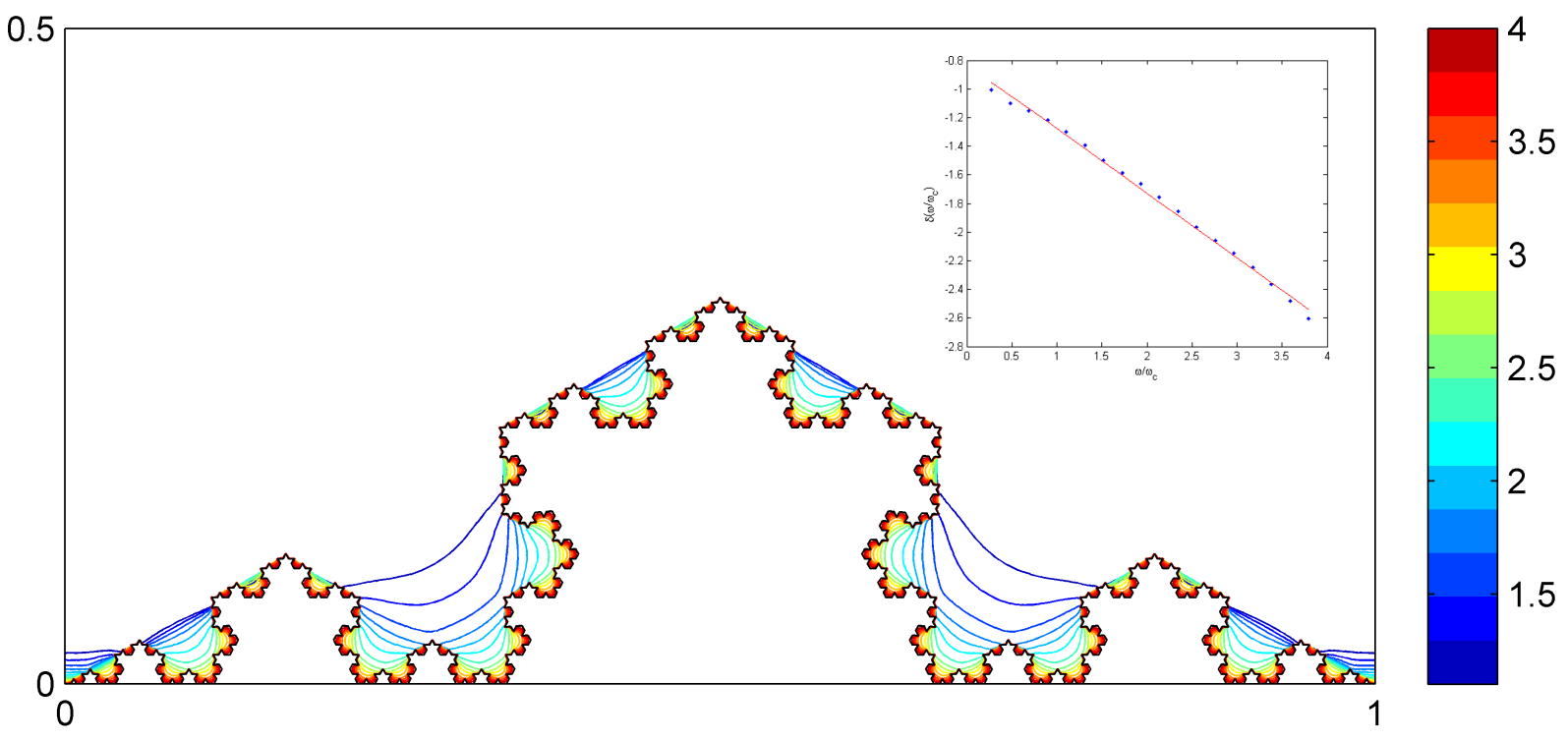

FIG. 5. Evolution of the boundary layer thickness, $\delta$, as a function of the frequency, $\tilde{\omega}$ for a fractal surface of dimension $d=1.2714$. Inset: scaling of the boundary layer thickness as a function of the nondimensional frequency. The scaling exponent for this fractal dimension equals -0.45 . 

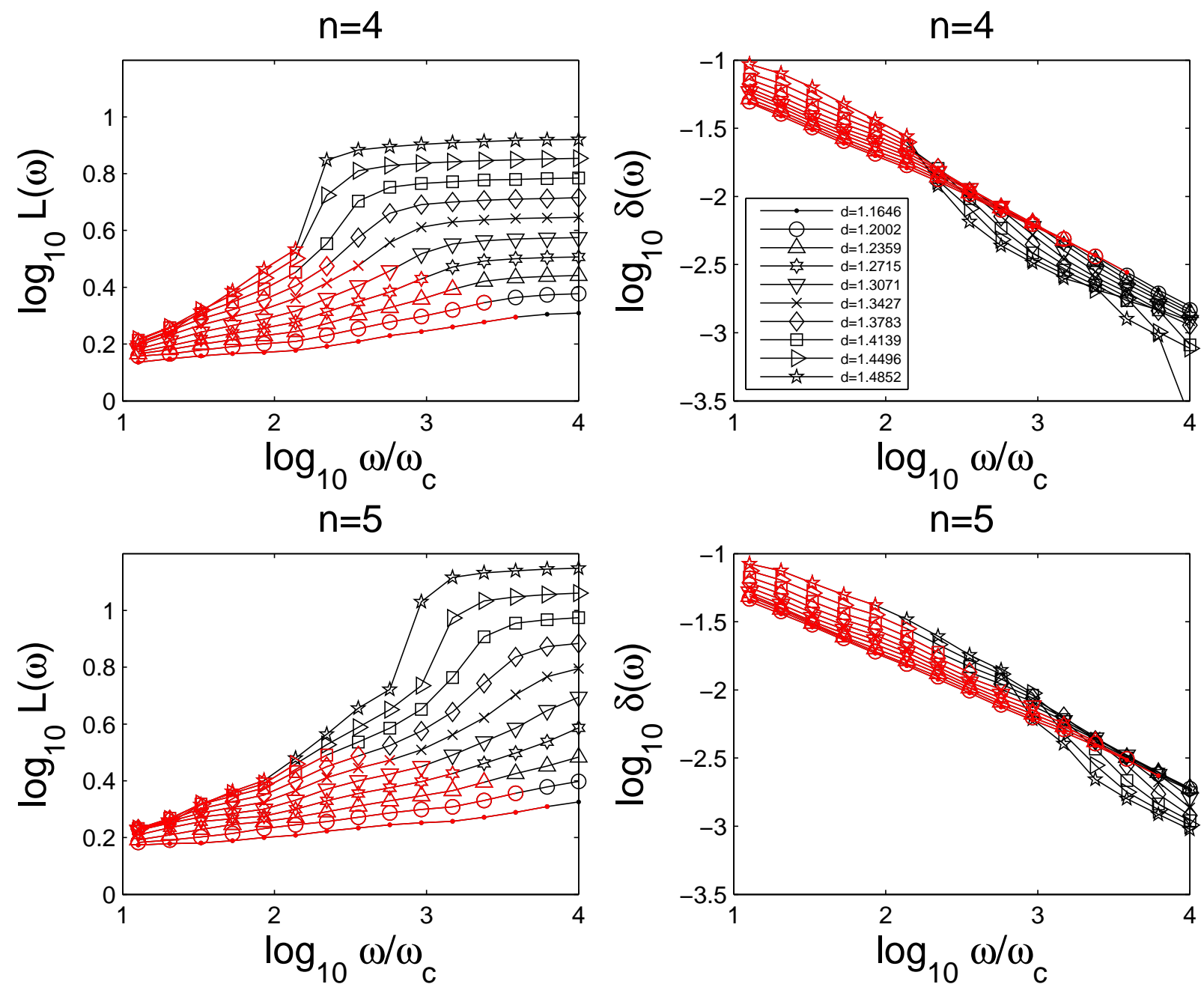

FIG. 6. Evolution of the length, $L(\omega)$, and thickness, $\delta(\omega)$, of the boundary layer for the entire range of fractal dimensions, $d$, and for a number of iterations in the construction of the fractal $n=4$ (top panels), and $n=5$ (bottom panels). 

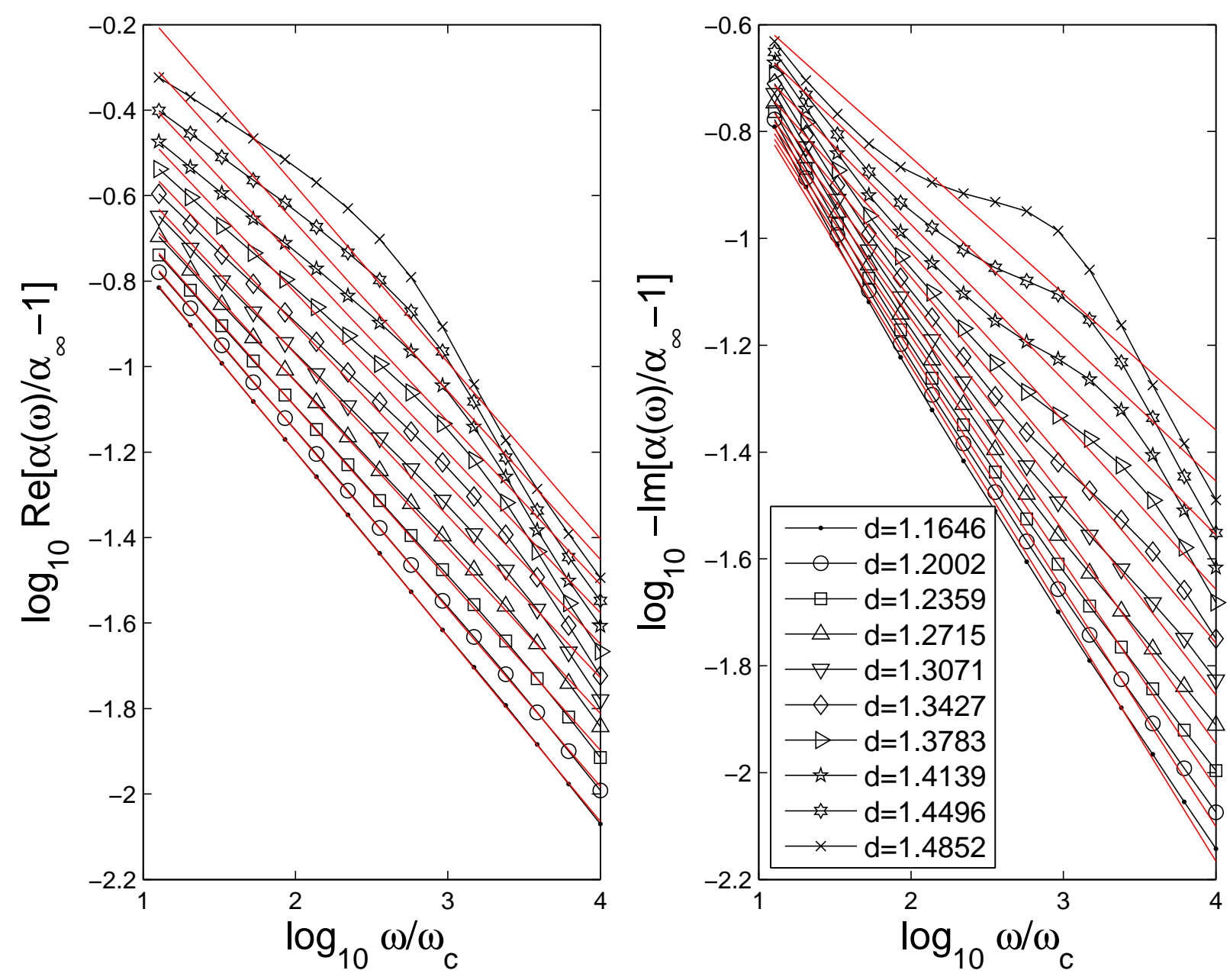

FIG. 7. Scaling of the real and imaginary parts of the added mass $\alpha(\omega) / \alpha_{\infty}-1$ for the range of fractal dimensions analyzed in this work. The red lines represent the best fit of (27) to the data. 

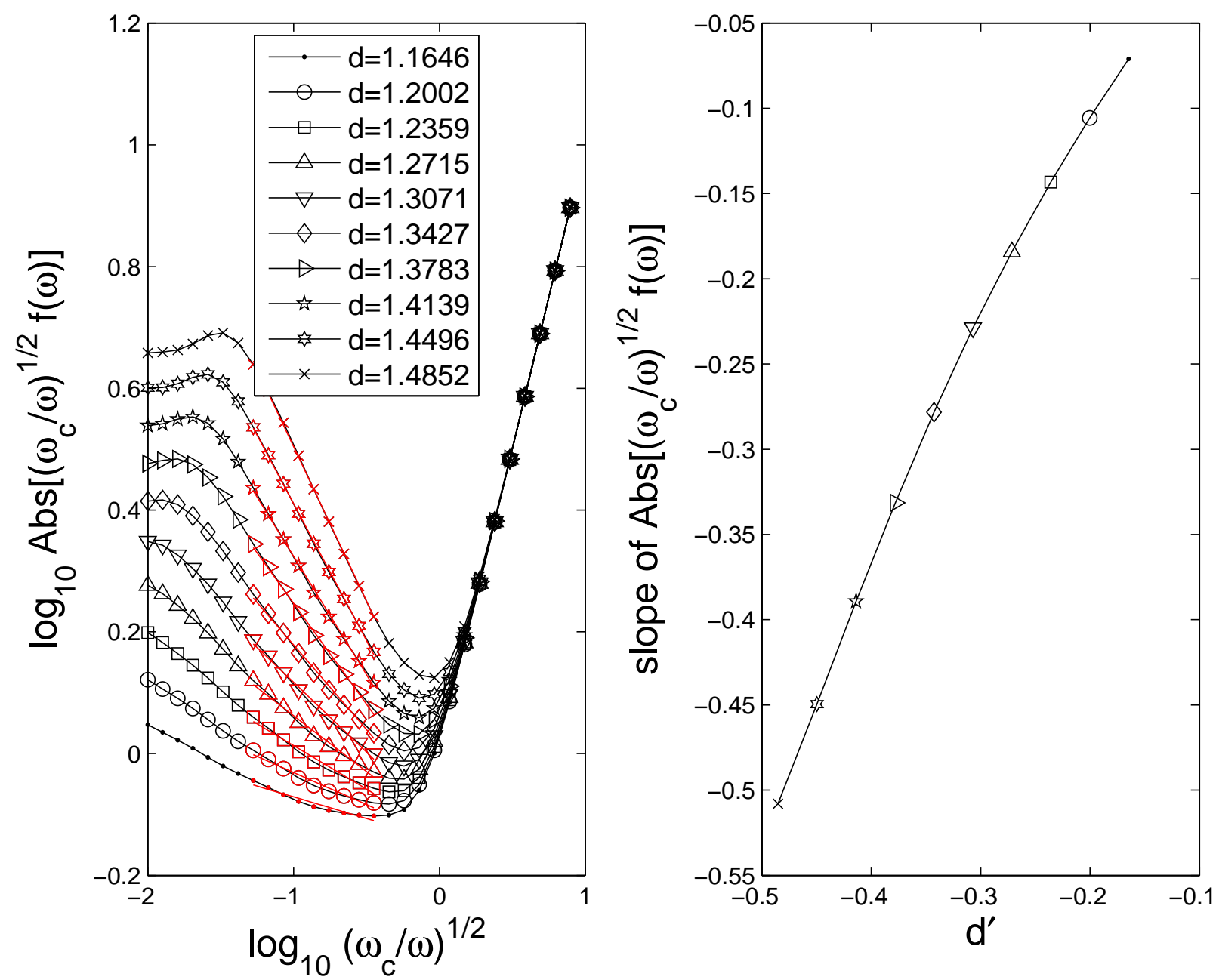

FIG. 8. Left hand side panel: Scaling of the drag force $f(\omega)$ absolute value, with the inverse of the square root of the nondimensional frequency $\omega / \omega_{c}$. Right hand side panel: comparison with Pozrikidis' model ${ }^{30}$, slope of $\left(\omega / \omega_{c}\right)^{(-1 / 2)} f(\omega)$ ] vs the $d^{\prime}$, the gain in wall length with increasing refinement. The best fit to the slope data points is a quadratic polynomial, $-1.4117 d^{\prime 2}+0.4544 d^{\prime}+$ 0.0423. Pozrikidis' model ${ }^{30}$ predicts that the slope and $d^{\prime}$ should be equal, whereas present results indicate deviations from this prediction. 

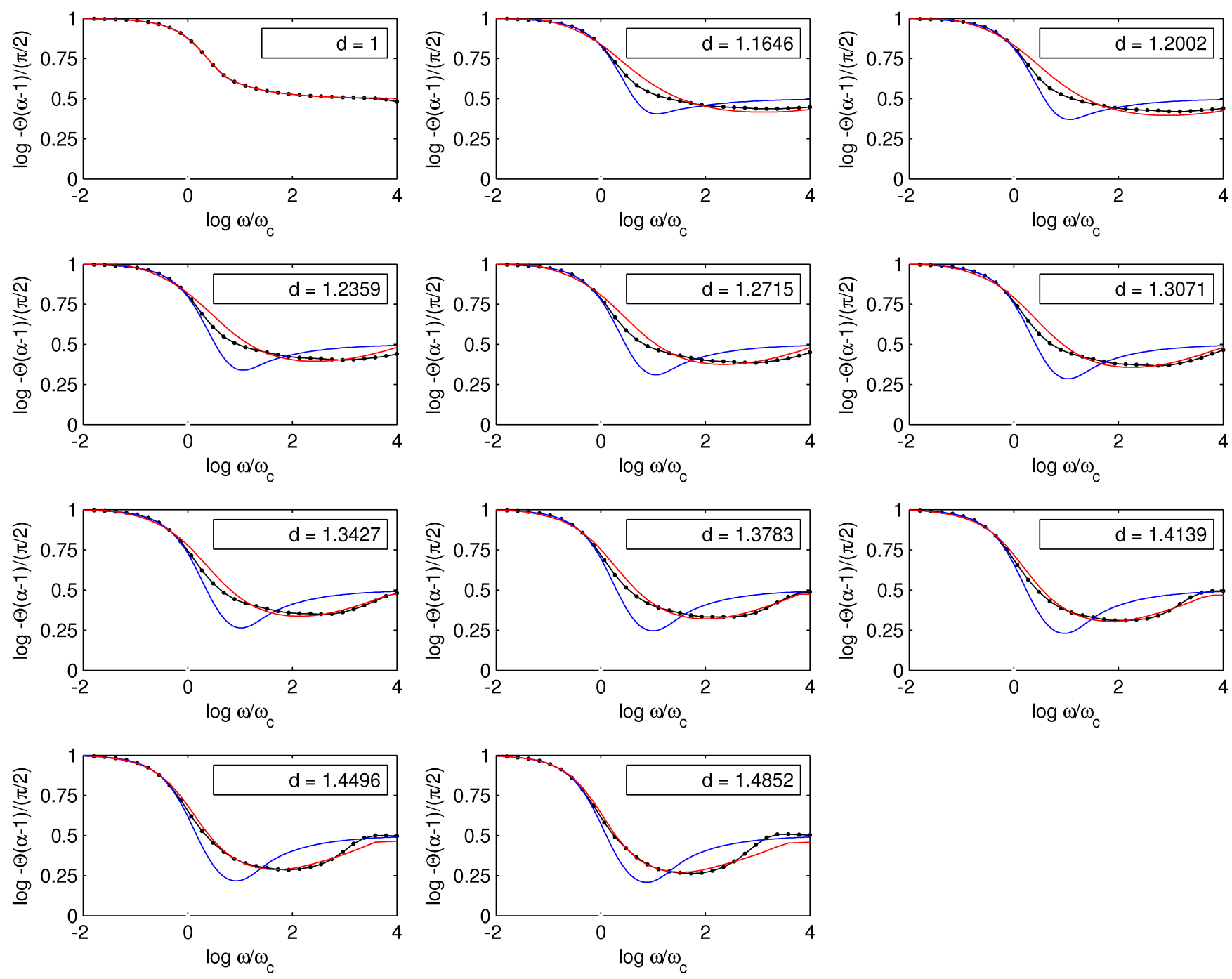

FIG. 9. Black dotted line: plot of the added-mass phase (normalized to $\pi / 2$ ) for all the fractal dimensions $d$ considered in this study. Blue line: best fit for the smooth-walls model [Eqs. (20), (23)]. Red line: best fit for the model proposed in this study [Eqs. (20), (29),(30), and (31)]. 


\section{DISCLAIMER}

This document was prepared as an account of work sponsored by the United States Government. While this document is believed to contain correct information, neither the United States Government nor any agency thereof, nor The Regents of the University of California, nor any of their employees, makes any warranty, express or implied, or assumes any legal responsibility for the accuracy, completeness, or usefulness of any information, apparatus, product, or process disclosed, or represents that its use would not infringe privately owned rights. Reference herein to any specific commercial product, process, or service by its trade name, trademark, manufacturer, or otherwise, does not necessarily constitute or imply its endorsement, recommendation, or favoring by the United States Government or any agency thereof, or The Regents of the University of California. The views and opinions of authors expressed herein do not necessarily state or reflect those of the United States Government or any agency thereof or The Regents of the University of California.

Ernest Orlando Lawrence Berkeley National Laboratory is an equal opportunity employer. 